\title{
Faint laser quantum key distribution: Eavesdropping exploiting multiphoton pulses
}

\author{
Stéphane Félix, Nicolas Gisin, André Stefanov and Hugo Zbinden*. \\ Group of Applied Physics, University of Geneva, 1211 Geneva 4, Switzerland \\ hugo.zbinden@physics.unige.ch, phone: +4122 702 68 83, fax: +4122 78109 \\ 80
}

(June 28, 2021)

\begin{abstract}
The technolgical possibilities of a realistic eavesdropper are discussed. Two eavesdropping strategies taking profit of multiphoton pulses in faint laser QKD are presented. We conclude that, as long as storage of Qubits is technically impossible, faint laser QKD is not limited by this security issue, but mostly by the detector noise.
\end{abstract}

\section{INTRODUCTION}

Quantum Cryptography or Quantum Key Distribution [1,2] offers provably secure key exchange [3]. Even for unlimited technological power of the eavesdropper, Eve, and technically limited devices of the legitimated users, Alice and Bob, the privacy of the exchanged key can be guaranteed.

In principle, single photons are sent from Alice to Bob. A quantum degree of freedom (e.g. polarization) is used to encode a bit value ( 0 or 1$)$. In practice, single photons are often mimicked by faint laser pulses containing on average much less than one photon per pulse. Hence, multiple photon pulses occur and open the door to efficient eavesdropping strategies that threaten the security of Quantum Key distribution, especially in combination with high losses in the fiber link [4]. In particular, suppose that:

i) due to losses in the fiber link, the fraction of pulses arriving at Bob links is smaller than the fraction of pulses containing more than one photon leaving Alice,

ii) Eve possesses a lossless fiber link from Alice to Bob,

iii) Eve can measure the number of photons without perturbing them (QND-measurement) and

iv) Eve can store photons without perturbing them for minutes.

In this case, Eve could measure the number of photons of the pulse. If there are less than two, she simply blocks the pulse. If there are more than one photon, she stores one of them and sends the other ones to Bob. After Bob and Alice announced the bases of the measurements, Eve measures her photons in the right bases and gets the full information. She does not create any errors and the average number of photons arriving at Bob remains unchanged.

In this paper we would like to discuss the security of faint laser QKD. We put emphasize on the multiphoton problem, considering realistic set-ups with nowadays technical limits and realistic eavesdroppers with state-of-art or near-future technology, but no unlimited technological power. For simplicity, we will restrict ourselves to the well-known 4-states protocol BB84 [1].

As you can imagine, the points ii) to iv) above are not easy to realize. There are no lossless fibers, no efficient QND-measurements and no efficient storage of photons for more than $\mu$ s nor transfer of the quantum degree of freedom to another quantum system like an ion in a trap. In section III we define the techniques a realistic Eve might possess. Nevertheless, using almost feasible techniques efficient eavesdropping is possible. Acting on two-photon pulses, Eve has two possibilities: First, she can apply an intercept-resend strategy making a measurement on both photons and sending a new photon to Bob according to the obtained result. In section IV we develop this strategy and calculate Eve's information per created bit error. Second, she can measure one photon and let pass the other one unchanged to Bob. In this case she won't introduce any errors, but she alters the photon statistics at Bob. In section V we calculate Eve's information as a function of the number of 2-photon pulses at Bob. In section VI, finally, we discuss the consequences of these eavesdropping strategies on the privacy amplification, the net bit rate and the maximal transmission distances.

In the next section we sum up the basics equations giving bit rates and photon number probabilities.

\section{TRANSMISSION RATES AND PHOTON STATISTICS.}

Alice produces coherent states with a low mean photon number $\mu$ which are only an approximation of single photon Fock-states. The probability that one finds $n$ photons in a coherent state follows the Poisson statistics:

$$
P(n, \mu)=\frac{\mu^{n}}{n !} e^{-\mu}
$$

In a second order approximation we obtain:

$$
\begin{gathered}
P(0) \approx 1-\mu+\frac{\mu^{2}}{2} \\
P(1) \approx \mu-\mu^{2} \\
P(2) \approx \frac{\mu^{2}}{2}
\end{gathered}
$$


Accordingly, the probability that a non-empty pulse contains more than 1 photon becomes:

$$
P(n \geq 2 \mid n>0)=\frac{1-P(0)-P(1)}{1-P(0)} \approx \frac{\mu}{2}+\left(\frac{\mu^{2}}{4}\right)
$$

The probability to have at least one photon is $1-P(1)=$ $1-e^{-\mu}$. Consequently, the probability to have a (single) count at Bob is:

$$
P_{\text {single }}=1-e^{-\mu t_{a b} \eta_{b}} \approx \mu t_{a b} \eta_{b}
$$

where $\mu$ has been replaced by $\mu t_{a b} \eta_{b}$, with $t_{a b}$ the transmission of the optical fiber and $\eta_{b}$ the detection efficiency of Bob's photon counter. The raw bit rate (before sifting, error correction and privacy amplification) is simply

$$
R_{\text {raw }}=v P_{\text {single }} \approx v \mu t_{a b} \eta_{b}
$$

with the pulse frequency $\nu$.

We can express $\mathrm{t}_{a b}$ in terms of the total loss $L_{a b}$ (in $\mathrm{dB}$ ), the product of $\alpha_{a b}$ (in $\mathrm{dB} / \mathrm{km}$ ) and $l_{a b}$, the length of the fibre in $\mathrm{km}$.

$$
t_{a b}=10^{-\frac{L_{a b}}{10}}=10^{-\frac{\alpha_{a b} l_{a b}}{10}}
$$

Eve's attacks must leave $P_{\text {single }}$ unchanged. Eve can make a beam-splitting attack (coupling out a fraction $\lambda$ ) and increase the fiber transmission up to $t_{e}$. Thus, she obtains:

$$
\lambda t_{e}=\lambda 10^{\frac{-\alpha_{e} l_{e}}{10}}=10^{\frac{-\alpha_{a b} l_{a b}}{10}}=t_{a b}
$$

Bob has two detectors per basis. Whenever he measures in a non-compatible basis, he has a certain probability to obtain a coincidence count. In the non compatible basis, the polarizing beamsplitter acts like a normal $50 \%$ coupler and we have on average $\frac{\mu}{2} t_{a b}$ photons in each arm. Accordingly the probability to have a click in both detectors becomes (the factor $\frac{1}{2}$ is for the probability to choose the wrong basis):

$$
P_{\text {coinc }}=\frac{1}{2}\left[1-e^{-\frac{\mu}{2} t_{a b} \eta_{b}}\right]^{2} \approx \frac{1}{8} \mu^{2} t_{a b}^{2} \eta_{b}^{2} \approx \frac{1}{4} P(2) t_{a b}^{2} \eta_{b}^{2}
$$

This is in first approximation equal to the intuitive calculation, taking simply the product of the probabilities to have two photons $P(2)$, the probability they arrive both at Bob $t_{a b}^{2}$, the probability that the non-compatible basis is chosen $\frac{1}{2}$, the probability that photons do not go to the same detector $\frac{1}{2}$, and finally the probability that both are detected $\eta_{b}^{2}$. (In the case of passive choice between the two bases, we find a factor of $\frac{5}{8}$ instead of $\frac{1}{4}$ ). Again, Eve must make sure that due to her intervention the number of coincidences is not changed.

\section{THE TECHNOLOGY OF A "REALISTIC" EVE}

In this paragraph, we present the technology at the disposal of a "realistic" Eve. We consider technolgies that are either state of the art or are imaginable with unlimited financial possibilities in the near future. It is important to note, that Eve can only take profit from the technologies available at the moment of key exchange. This is in contrast to the public key system, where messages that should remain secret for a long time are also threatened by future technologies. For simplicity, in some cases when there is no important consequence for the efficiency of her attacks, we attribute to Eve perfect technology.

\section{A. Eve's infrastructure and optical components}

Eve has free access to Alice and Bob's quantum channel at any point outside their offices. She can install optical components (switches, couplers etc.) without being noticed. Eve's components have no losses and no backscattering and are therefore invisible. Her optical switches are arbitrarily fast. In particular, she can in arbitrary short times recover the original situation whenever Alice and Bob check their line with an OTDR (optical time domain reflectometer). She knows Alice and Bob's bases and can perfectly align her analyzers with respect to these bases].

\section{B. Eve's photon counters}

Eve's photon counters are perfect. They feature a quantum efficiency of 1 and no noise. Moreover, they can count photons arriving at the same time. Hence, Eve can in particular distinguish between pulses containing one and two photons, but the measurement is destructive (no QND).

\section{Eve's fibre link}

Eve doesn't have lossless fibres. Nowadays telecom fibres have reached a such a high quality level that the losses in the second telecom window (@1550 nm) are close to the theoretical limit. This means that a value of $0.20 \mathrm{~dB} / \mathrm{km}$ corresponds to the losses you expect from

\footnotetext{
${ }^{1}$ This is trivial in the cases where Alice and Bob use strong classsical pulses to align their setups. In the auto-aligned "plug\&play" setup 12, the phase difference between the two pulses is constantly fluctuating due to thermal instabilities. Bob could even introduce a random phase for each bit, rendering the task of Eve even more difficult.
} 
inevitable Rayleigh backscattering due to the different molecules (essentially silica and germanium) present in the glass. Fibers with a pure silica core have lower losses (at the moment down to $0.171 \mathrm{~dB} / \mathrm{km}$ [5]) but are actually not telecom standard. In contrary, real fibres have additional losses due to splices and connections in the centrals and a value of $0.25 \mathrm{~dB} / \mathrm{km}$ is more realistic. Moreover, installed fibres rarely follow the bee-line $\left(d_{a b}\right)$ (they often follow roads and go from one telecom central to another). We assume that Eve possesses a fiber link relating Alice and Bob on a straight line with a loss $\alpha_{e}$ of $0.15 \mathrm{~dB} / \mathrm{km}$. Alice and Bob must measure the total loss $L_{a b}$ of their link. Eve's maximum transmission gain $G_{t}$ $\left(G_{t}=10 \log \left(\frac{t_{e}}{t_{a}}\right)\right)$ is then $L_{a b}-\alpha_{e} d_{a b}$. If the time of flight of the photons is monitored, $G_{t}$ is only $\left(\alpha_{a b}-\alpha_{e}\right) l_{a b}$, which is according to our assumptions $\approx 0.1 \mathrm{~dB}$ per fibre $\mathrm{km}$. At any other wavelength $G_{t}$ would be larger.

Note that Eve could introduce in advance additional loss between Alice and Bob. Crypto users must therefore calculate the loss $L_{a b}$ and $\alpha_{a b}$ of their line from the obtained $P_{\text {single }}$ and compare it to the usual values?. The higher the accepted loss is, the higher $G_{t}$, and as we will see, the higher Eve's information.

\section{Eve's photon sources}

Eve possesses perfect single photon sources and more general sources that can emit arbitrary states at demand. Nowadays, such kind of sources are far from being realized. However, admitting such sophisticated sources has not much impact on the security and performance of practical QKD systems, since in most cases only the single photon and two-photon statistics can be measured by Bob.

\section{E. Eve's possibilities to modify and influence Alice and Bob's apparatuses}

In cryptography in general, Alice and Bob's offices have to be supposed to be secure. We also assume that Eve cannot manipulate Alice and Bob's crypto devices before they get installed (or that they are quantum physicist that can test them). This is not trivial, and in practice crypto users have to trust their suppliers. However, due to the optical fiber coming into the offices Alice and Bob have to be aware of, what is sometimes called "Trojan horse" attacks. In such kind of attacks Eve sends light pulses at arbitrary wavelengths and analyzes the reflected light in order to find out e.g. which detector clicked or which phase was applied. We assume that Alice and

\footnotetext{
${ }^{2}$ Note that a standard measurement of the fiber transmission could be manipulated by Eve.
}

Bob applied all precautionary measures to prevent such strategies. In the same line, we take it for granted that Alice and Bob do not send any parasite signals that could reveal their bits. The problem is not that these counter measures are technically difficult to realize, but the prove that all leaks have been plugged up.

All these assumptions are essential in the sense that otherwise secure QKD is not possible. Another assumption commonly made is that all bit errors detected by Alice and Bob are introduced by Eve, and that she possesses the corresponding information of the key. This means that privacy amplification has to be applied to reduce this potential information, which in turn, in the cases of large quantum bit error rates (QBER), drastically reduces the bit rate. In practice also without any eavesdropping, QKD-systems suffer from considerable QBER, that has two origins [6]. A first part, $\mathrm{QBER}_{\text {opt }}$, is due to non perfect alignment of the optical setup e.g. the polarization measurement bases of Alice and Bob are not perfectly parallel, or the light pulses are not perfectly polarized. The second, and mostly dominant, part is due to darkcounts of the photon counters. Attributing all QBER to the presence of an eavesdropper and assuming that Eve gains the corresponding information (see section III), means supposing that Eve has ways to reduce $\mathrm{QBER}_{\text {opt }}$ and $\mathrm{QBER}_{\text {det }}$. Only diminishing these errors allows her to tap the line and gain information without introducing additional errors. On the one hand, one can imagine that Eve can reduce $\mathrm{QBER}_{\text {opt }}$ in certain cases, by improving e.g. the alignment of Alice and Bob's bases. One the other hand, reducing $\mathrm{QBER}_{\text {det }}$ i.e. the dark count rate at a distance by sending some magic light pulses is not conceivable. Therefore, we suggest that only for the difference between the measured QBER $\left(\mathrm{QBER}_{m e s}\right)$ and the presumed $\mathrm{QBER}_{\text {det }}$ privacy amplification must be applied. Typically, $\mathrm{QBER}_{o p t}$ is in the order $0.5 \%$. Thus, adding a tolerance of $0.5 \%$, we may attribute an error of $1 \%$ to Eve.

Similarly, we assume that Eve cannot increase the detection efficiencies of Bob's detectors. This would be very useful for the eavesdropping strategy discussed in section IV. Instead of lowering the losses in the quantum channel, Eve could then simply increase the efficiency of the detectors (for the pulses, from which she was able to extract one photon), as suggested by Lütkenhaus [4].

\section{STRATEGY A: INTERCEPT-RESEND WITH MULTI-PHOTON PULSES}

Eve intercepts all photons and analyzes them with the setup shown in Fig. 1. She transmits the measurement results through a (lossless) classical channel to a source close to Bob that sends the most suitable states, respecting the photon statistics expected by Bob. Eve sends the photons via passive beamsplitter to both measurement bases. Consequently, she obtains four different possible 
results (cases with more than 2 photons are neglected). In table 1 the four different possibilities are listed with the corresponding probabilities $\mathrm{p}$ (the conditional probability, provided that at least one photon is detected), Eve's information per bit $I(A, E)$, the QBER she creates and the ratio of information per QBER. The case A (one photon) corresponds to the standard interceptresend on single photon pulses [7]. Case B, two photons, each in a different basis, is the most favorable one for Eve. First, after the sifting process she will possess the full information on the bit value. Second, by sending an intermediate state, a linear combination of the two corresponding states separated by $\frac{\pi}{4}$, she will create a smaller error (0.15) than in case A. Case $\mathrm{C}$, both photons in the same detector, is still favorable. In contrary, Eve will discard if possible all events $\mathrm{D}$, two photons is the same (wrong) basis, where she gains no information at all.

\begin{tabular}{|l|c|c|c|c|}
\hline case & $p$ & $I(A, E)$ & $Q B E R$ & $\frac{I(A, E)}{Q B E R}$ \\
\hline $\mathrm{A}$ & $1-\frac{\mu}{2}$ & $\frac{1}{2}$ & $\frac{1}{4}$ & 2 \\
\hline $\mathrm{B}$ & $\frac{1}{2} \frac{\mu}{2}$ & 1 & $\sin ^{2}\left(\frac{\pi}{8}\right)=0.15$ & 6.83 \\
\hline $\mathrm{C}$ & $\frac{3}{8} \frac{\mu}{2}$ & $\frac{2}{3}$ & $\frac{1}{6}$ & 4 \\
\hline $\mathrm{D}$ & $\frac{1}{8} \frac{\mu}{2}$ & 0 & $\frac{1}{2}$ & 0 \\
\hline
\end{tabular}

Of course, Eve has to make sure that the number of photons arriving at Bob remains unchanged. If the transmission of Alice and Bob's quantum channel is lower than the probability of getting case B, Eve will only send photons in this case. If this outcome is not abundant enough, she will have to add pulses after detections of kind $\mathrm{C}$ and finally of kind A and possibly even B. This is illustrated in Fig. 2, where Eve's information per bit error is given as a function of fiber length. One can notice that from a distance of about $65 \mathrm{~km}$ on, Eve can entirely rely on case $\mathrm{B}$ and obtains therefore 3.4 times more information per bit error than in the case A (usual intersept-resend) that applies in the limit of very short fibers.

Eve pays attention to reproduce the same number of coincidences at Bob, which is no problem according paragraph III D.

\section{STRATEGY B: MEASURING ONE PHOTON OUT OF TWO IN A PULSE}

The easiest way to take profit of two photons in a pulse is to couple a fraction $\lambda$ out of each pulse with a beamsplitter and replace the original line with a line that has lower loss, in order to compensate for $\lambda$ (see Fig. 3). Then clearly, the number of photons (and their statistics) arriving at Bob remains unchanged and this attack does not generate any errors. Nevertheless, Eve gains the following amout of information:

$$
I(A, E)=\frac{\mu}{2}(2 \lambda(1-\lambda)) \frac{1}{2}
$$

where $\frac{\mu}{2}$ is the probability to find two photons in a pulse, $2 \lambda 1-\lambda$ is the probability that these two photons sep- arate at the coupler (outcoupling probability of $\lambda$ ) and finally the outcoupled photon has a probability of $\frac{1}{2}$ to end up in the right basis. We can see immediately that this information is maximum $\left(I(A, E)=\frac{\mu}{8}\right)$ for $\lambda=\frac{1}{2}$, corresponding to a gain $G_{t}$ of $3 \mathrm{db}$. If (instead of only one beamsplitter) many couplers and analyzers are used in series, with a total coupling loss that equals $G_{t}$, the information can go up to $\frac{\mu}{4}$. This value can be approached for $G_{t} \gtrsim 6 d b$.

In order to further increase her information, Eve must favor the detection of two photon pulses. She introduces therefore a shutter (see Fig. 3) that allows her to block a fraction $(1-\gamma)$ of the pulses, when she didn't catch one photon. In this way the photon distribution is no longer Poissonian. The calculation of the photon distribution at the entrance port of Bob, as a function of $\lambda$ and $(1-\gamma)$, is straightforward but lengthy. The probabilty for pulses containing $\mathrm{n}$ photons $(\mathrm{n}>0)$ is

$$
P^{\prime}(n, \lambda, \gamma)=\frac{1}{n !}(1-\lambda)^{n} \mu^{n} t_{e}^{n}\left[\begin{array}{c}
(\gamma-1) e^{-\mu+(1-\lambda)\left(1-t_{e}\right) \mu} \\
+e^{-\mu(1-\lambda) t_{e}}
\end{array}\right]
$$

with the transmission of Eve's fiber $t_{e}$. With $P_{\text {single }}^{\prime}(\lambda, \gamma) \approx \eta_{b} P^{\prime}(1, \lambda, \gamma)$ we obtain for Bob's singles count probability :

$$
P_{\text {single }}^{\prime}(\lambda, \gamma) \approx(1-\lambda) \mu t_{e} \eta_{b}\left[\begin{array}{c}
(\gamma-1) e^{-\mu+(1-\lambda)\left(1-t_{e}\right) \mu} \\
+e^{-\mu(1-\lambda) t_{e}}
\end{array}\right]
$$

This formula corresponds approximatively, to what we obtain by simply adding up the probabilities for 1- and 2-photon pulses:

$$
\begin{aligned}
P_{\text {single }}^{\prime}(\lambda, \gamma) & \approx t_{e} \eta_{b}\left[\begin{array}{c}
2(1-\lambda) \lambda P(2) \\
+\gamma\left((1-\lambda) P(1)+2(1-\lambda)^{2} P(2)\right)
\end{array}\right] \\
& \approx(1-\lambda) \mu t_{e} \eta_{b}[\lambda \mu+\gamma((1-\mu)+(1-\lambda) \mu)]
\end{aligned}
$$

The first term (inside the square brackets) is the favorable case, when there are two photons in the pulse and one of them goes to the detectors. The second and the third term correspond to 1 and 2 photons propagating to Bob, respectively. This value must equal to $P_{\text {single }}$ given in eq. [5] without spy. which is the e.g. case for $\gamma=1$ when $t_{a b}=\frac{t_{e}}{2}$ and $\lambda=\frac{1}{2}$. This case correspond to the simple attack with a $3 \mathrm{db}$ coupler, presented at the top of this section.

Eve can block all pulses without detection $(\gamma=0)$, as soon as $t_{a b} \leq \frac{t_{e} \mu}{4}$. This is the case for $\mu=0.1$ when $G_{t} \geq 16 d B$. Then, Eve get's $50 \%$ of the information without creating errors. This simple attack is almost as efficient as an attack based on a complicated QND measurement, where same result is obtained for $t_{a b} \leq \frac{t_{e} \mu}{2}$. If Eve had at disposition some device for storing photons, she could wait for the sifting process, then measure her photons in the right basis and get 100\% information. In 
consequence, this kind of attacks are very powerful as soon Eve's $G_{t}$ is large.

However, by acting selectively on two-photon pulses Eve changes the photon statistics and her presence might be revealed by Bob's coincidence measurements. According to eq. [9] Bob's coincidence rate is $P_{\text {coinc }} \approx \frac{1}{8} \mu^{2} t_{a b}^{2} \eta_{b}^{2}$. On the other hand with $P_{\text {coinc }}^{\prime}(\lambda, \gamma) \approx \frac{1}{4} \eta_{b}^{2} P^{\prime}(2, \lambda, \gamma)$ we get:

$$
P_{\text {coinc }}^{\prime}=\frac{1}{8}(1-\lambda)^{2} \mu^{2} t_{e}^{2} \eta_{b}^{2}\left[\begin{array}{c}
(\gamma-1) e^{-\mu+(1-\lambda)\left(1-t_{e}\right) \mu} \\
+e^{-\mu(1-\lambda) t_{e}}
\end{array}\right]
$$

In order to fulfill the two conditions

$$
\begin{aligned}
P_{\text {single }}^{\prime} & =P_{\text {single }} \\
P_{\text {coinc }}^{\prime} & =P_{\text {coinc }}
\end{aligned}
$$

Eve can vary the parameters $\gamma$ and $\lambda$. But we realize that for $\gamma \neq 1$, Eve cannot satisfy both conditions. For equal single counts, she always increases the number of coincidences detected by Bob. If the effect of Eve's interaction was to reduce the coincidence count rates, she could add from time to time photons at price of additional errors. But reducing the number of coincidences without changing the bit rate, is impossible with linear optics (i.e. without QND-measurement of the photon number). However, the number of detected coincidences is small and statistical fluctuation are large. In Fig. 4 we see Bob's coincidence counts as a function of $\gamma$, for $10^{10}$ pulses sent over $60 \mathrm{~km}$. The horizontal lines are the coincidence rates without Eve $\left(P_{\text {coinc }}\right)$, and $\pm 2 \sigma\left(P_{\text {coinc }} \pm 2 \sqrt{P_{\text {coinc }}}\right)$ variations. In the same graph Eve's information is plotted, too. We can readout the information Eve obtains, by increasing Bob's coincidence counts by $2 \sigma . I(A, E)$ is given by

$$
\begin{aligned}
I(A, E) & =\gamma \frac{\mu}{2} \frac{1}{2} 2 \lambda(1-\lambda)+(1-\gamma) \frac{1}{2} \\
& =\gamma \frac{\mu}{2} \lambda(1-\lambda)+(1-\gamma) \frac{1}{2}
\end{aligned}
$$

where the two terms are Eve's information for a nonactivated shutter (according eq. (10) ) and for an activated shutter $\left(\frac{1}{2}\right)$, respectively.

\section{IMPACT OF MULTIPHOTON EAVESDROPPING ON BITRATES AND MAXIMUM TRANSMISSION DISTANCE}

After the sifting process Alice and Bob correct the errors in their raw key and apply privacy amplification in order to reduce Eve's information to an arbitrarily low level. The efficiency of these processes depends of course on the QBER and $\mathrm{I}(\mathrm{A}, \mathrm{E})$, respectively $[10, ?]$. So high error rates have their consequence on the net bit rate
$R_{n e t}$ (or distilled bit rate $R_{d i s t}$ ), which is at the end, together with the transmission distance, the the only figure of merit of QKD. Therefore it is helpful to plot $R_{n e t}$ as a function of fiber length. $R_{n e t}$ decreases in a first time exponentially (linearly in log plot) due to the fiber losses, before at a certain point it rapidly falls to zero due to the exploding losses due to the necessary error correction and privacy amplification. This point, representing the maximum transmission distance, depends now on the estimation of Eve's information corresponding to her technical possibilities. In practice, nowadays systems are essentially limited by the detector noise, which leads to high QBER at large distances. In order to make the impact of the discussed eavesdropping strategies more transparent, and to estimate the limits of future systems with improved detectors, we assume in the following graphs a dark count probability of $10^{-6}$ instead of the actually typical $10^{-5}[9]$. We compare the impact of the presented strategies with the scenario of an Eve with unlimited technological power as proposed by Lütkenhaus. The Lütkenhaus curve is dropping down rapidly, because whenever $t_{a b} \eta_{b} \approx \frac{\mu}{2}$ Eve's information $I(A, E) \approx 1$ and almost everything of the key is lost by privacy amplification. Therefore the curve is calculated with an optimal $\mu$ which decreases with the transmission distance (loss). This has in turn the same effect, that the $R_{n e t}$ is dropping down. .

In Figure 5, we see $R_{n e t}$ as a function of distance for strategy A for different values of $\mu$. We assume Eve's induced error rate to be $Q B E R_{\text {mes }}-Q B E R_{\text {det }} \approx 1 \%$, and $I(A, E)$ corresponding to this error has to be corrected. The maximum transmission distance is not considerably reduced with respect to an analog curve without multiphoton attacks, or to the shown curve without eavesdropper (no privacy amplification). The reason is that in the worst case (case B) we have to correct $6.8 \%$ of information instead of $2 \%$ for single photon intercept-resend. This means we will approximately loose $6.8 \%$ of the key instead of $2 \%$. However, the bit loss due the error correction is in comparison much more important. So we can say for strategy A, multiphoton-pulses do not reduce the maximum transmission loss, nevertheless we have to be aware of the problem and apply the corresponding privacy amplification.

Figure 6 shows the result for strategy B. We suppose that $I(A, E)$ corresponding to a $2 \sigma$ increase of $P_{\text {coinc }}^{\prime}$ has to be corrected. The difference with respect to the shown curve without eavesdropping is small 3. $R_{n e t}$ is not considerably reduced since the maximum $I(A, E)$ is $\frac{1}{2}$, which reduces $R_{n e t}$ by a factor of 2 . This is illustrated by the

\footnotetext{
${ }^{3}$ This would be the same for lossless fibers $\left(\alpha_{e}=0 d B\right)$ since $\gamma$ is not limited by the $G_{t}$ but by the change in $P_{\text {coinc }}^{\prime}$ (see Fig. 4).

${ }^{4}$ QKD-systems that do not monitor $P_{\text {coinc }}$ must systemati-
} 
curve where photon storage is admitted and $I(A, E)$ can go up to 1 , and therefore $R_{n e t}$ drops down faster. However, the transmission distance is only dramatically reduced, if photon storage is combined with conservation of photon statistics, which asks for QND-measurements. Therefore under realistic conditions, even if strategy $\mathrm{B}$ is more efficient than strategy A, it does not limit the transmission of faint laser QKD.

Our calculation give for both eavesdropping strategies higher $R_{n e t}$ for higher $\mu$. For strategy $\mathrm{B}, I(A, E)$ decreases with a increasing $\mu$, since $\frac{2 \sqrt{P_{\text {coinc }}}}{P_{\text {coinc }}}$ decreases and Eve's presence is discovered faster. However, our calculation for strategy $\mathrm{A}$ is only correct for $\mu \ll 1$, indeed, it becomes much more powerful for $\mu \approx 1$. We plan to study the efficiency of this strategy for larger $\mu$, in order to optimize $\mu$ and hence the bit rate of faint laser QKD.

\section{CONCLUSIONS}

We have shown that also with realistic technologies an eavesdropper can easily take profit of multi-photon pulses. However, the impact on the maximum transmission distance is minor. In order to guarantee this, Bob must survey the length of the fiber, its loss and the coincidence count rates in order to limit Eve's information. We think it is not reasonable to attribute all QBER to Eve. Along the same line, in the case of increased QBER, it might be better to check the causes, instead of simply perform error correction and privacy amplification.

It is important to note, that QND-measurements alone, does not allow dangerous multi-photon attacks. The crucial point is whether Eve can store photons or not. One can take for granted that it is impossible to store millions of qubits efficiently for seconds in the near future. Users who worry about that may just wait a few minutes before proceeding with sifting. Therefore for the time being, faint laser QKD has no security problem due to multiphoton pulses. It is limited in distance by the dark counts of the detectors. In conclusion, QKD using entangled photons is not mandatory 8,13 .

\section{ACKNOWLEDGMENTS}

We thank Norbert Lütkenhaus and François Cochet for helpful discussions. This work was supported by the

cally correct for the maximum $I(A, E)$ for a given distance. The fact of measuring the $P_{\text {coinc }}$ can increase $R_{\text {net }}$ by a factor of up to 2 .

${ }^{5}$ Note, that QKD-setups based on parametric downconversion face an identical multi-photon problem, if the choice of the bases is not passive [8].
Esprit project 28139 (EQCSPOT) through Swiss OFES and the FNRS.

\section{REFERENCES}

[1] Ch.H. Bennett, and G. Brassard, "Quantum cryptography: public key distribution and coin tossing", Int. conf. Computers, Systems \& Signal Processing, Bangalore, India, December 10-12, 175-179 (1984).

[2] N. Gisin, G. Ribordy, W. Tittel, and H.Zbinden, "Quantum Cryptograpy", quant-ph/0101098, submitted to Rev. Mod. Phys. (2000).

[3] D. Mayers, "Unconditional security in quantum cryptography", Journal for the Association of Computing Machinery (to be published)(1998); also in quantph/9802025; H.-K. Lo and H.F. Chau, "Unconditional security of quantum key distribution over arbitrary long distances" Science 283, 2050-2056 (1999); P.W. Shor, and J. Preskill, "Simple proof of security of the BB84 Quantum key distribution protocol", Phys. Rev. Lett. 85, 441 (2000)

[4] N. Lütkenhaus, "Security against individual attacks for realistic quantum key distribution", Phys. Rev. A, 61, 052304 (2000).

[5] T.Kato, M. Hirano, M. Onishi, and M. Nishimura, "Ultra-low nonlinearity low-loss pure silica core fibre for long-haul WDM transmission,, Electron. Lett. 35 (19), 1615-1617 (1999).

[6] H. Zbinden, H. Bechmann-Pasquinucci, N. Gisin, G. Ribordy, "Quantum Cryptography", Appl. Phys. B 67, 743-748 (1998).

[7] Ch.H. Bennett, F. Bessette, G. Brassard, L. Salvail, and J. Smolin, "Experimental Quantum Cryptography", J. Cryptology 5, 3-28 (1992).

[8] G.Ribordy, J. Brendel, J.D. Gautier, N. Gisin, and H. Zbinden, "Long distance entanglement based quantum key distribution", quant-ph 0008039, Phys. Rev. A 63, 012309 (2001)

[9] D. Stucki, G. Ribordy, A. Stefanov, H. Zbinden, J.G. Rarity, T. Wall, "Photon counting for quantum key distribution with Peltier cooled InGaAs APD's", J. Mod. Opt, this issue (2001)

[10] G. Brassard, and L. Salvail, "Secret-key reconciliation by public discussion", in Advances in Cryptology, Eurocrypt '93, Lecture Notes in Computer Science 765, 410-423 (1993).

[11] Ch.H. Bennett, G. Brassard, C. Crepeau, and U.M. Maurer, "Generalized privacy amplification", IEEE Trans. Information th., 41, 1915-1923 (1995).

[12] G. Ribordy, J.-D. Gautier, N. Gisin, O. Guinnard, H. Zbinden, "Fast and user-friendly quantum key distribution", J. Mod. Opt., 47, 517-531(2000).

[13] G. Brassard, N. Lütkenhaus, T. Mor, and B.C. Sanders, 
"Limitations on Practical Quantum Cryptography", Phys. Rev. Lett. 85, 1330-1333 (2000).

\section{FIGURE CAPTIONS}

Fig 1: Eve's setup for strategy A: $\mathrm{S}=$ optical switch, $\mathrm{PBS}=$ polarization beam splitter, $\mathrm{C}=$ photon counter

Fig 2: Strategy A: Eve information $I(A, E)$ according to the different regimes (see table 1) as function of distance, for $\alpha_{a b}=0.25 \mathrm{~dB} / \mathrm{km}$ and $\mu=0.1$.

Fig 3: Eve's setup for strategy B. $\mathrm{S}=$ optical switch, $\mathrm{PBS}=$ polarization beam splitter, $\mathrm{C}=$ photon counter

Fig. 4: Strategy B: Eve's information $I(A, E)$ and number of coincidences as a function of $\gamma$, under the condition of unchanged single counts $\left(P_{\text {single }}^{\prime}=P_{\text {single }}\right)$. The parameters are: $\mu=0.1$, number of pulses $10^{10}$, $l_{a b}=60 \mathrm{~km}, \alpha_{a b}=0.25 \mathrm{~dB} / \mathrm{km}, \alpha_{e}=0.15 \mathrm{~dB} / \mathrm{km}$

Fig. 5: Strategy A: Normalized $R_{n e t}$ as a function of distance for different values of $\mu$. For comparison curves with no eavesdropper (only error correction applied) and an eavesdropper with unlimited technology (Lütkenhaus) are shown.

Fig. 6: Strategy B: Normalized $R_{n e t}$ as a function of distance. The parameters are: $\mu=0.1$, number of pulses $10^{10}, l_{a b}=60 \mathrm{~km}, \alpha_{a b}=0.25 \mathrm{~dB} / \mathrm{km}, \alpha_{e}=0.15 \mathrm{~dB} / \mathrm{km}$. For comparison curves with no eavesdropper (only error correction applied) an eavesdropper with storage device and an eavesdropper with unlimited technology (Lütkenhaus) are shown. 


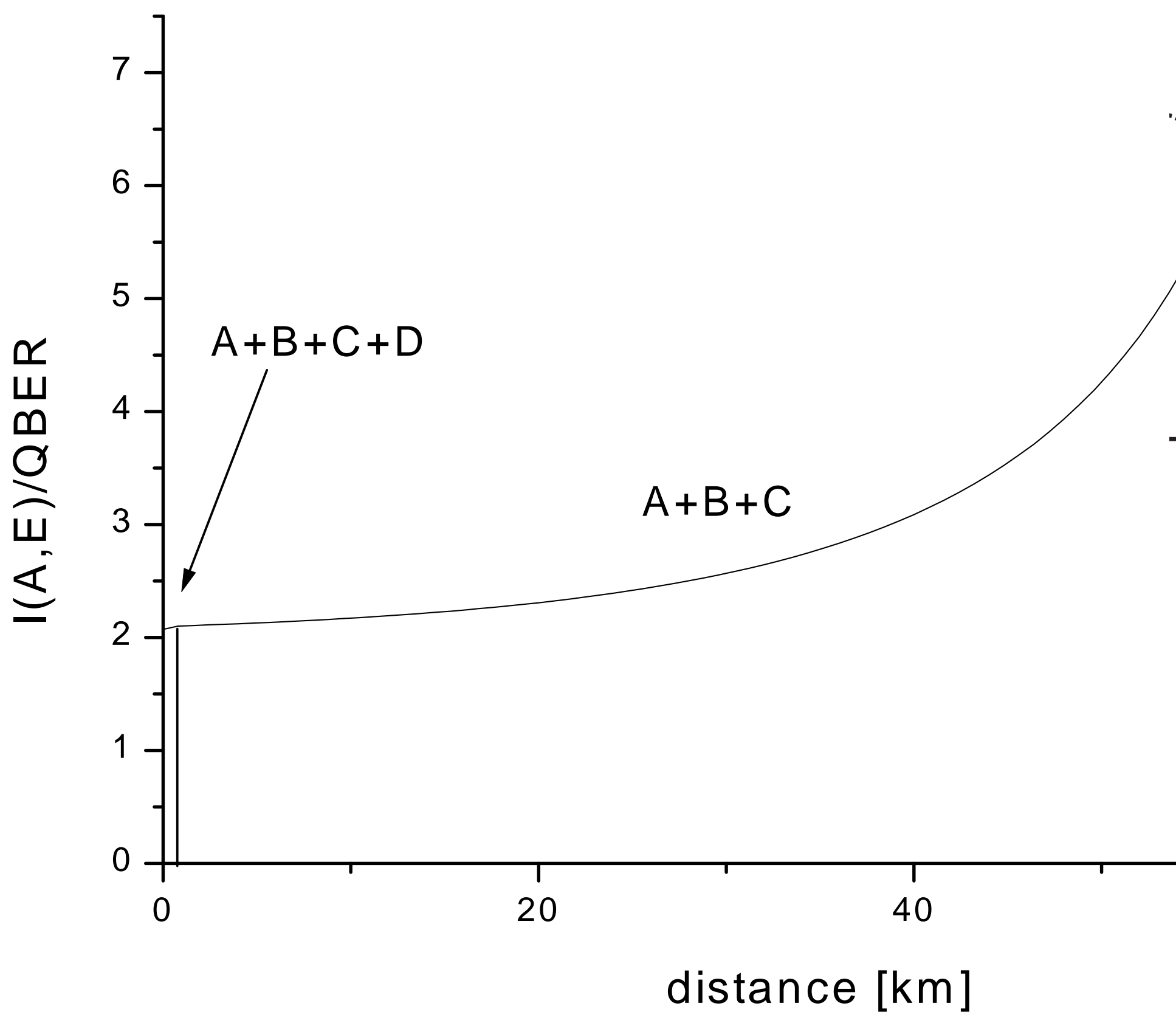




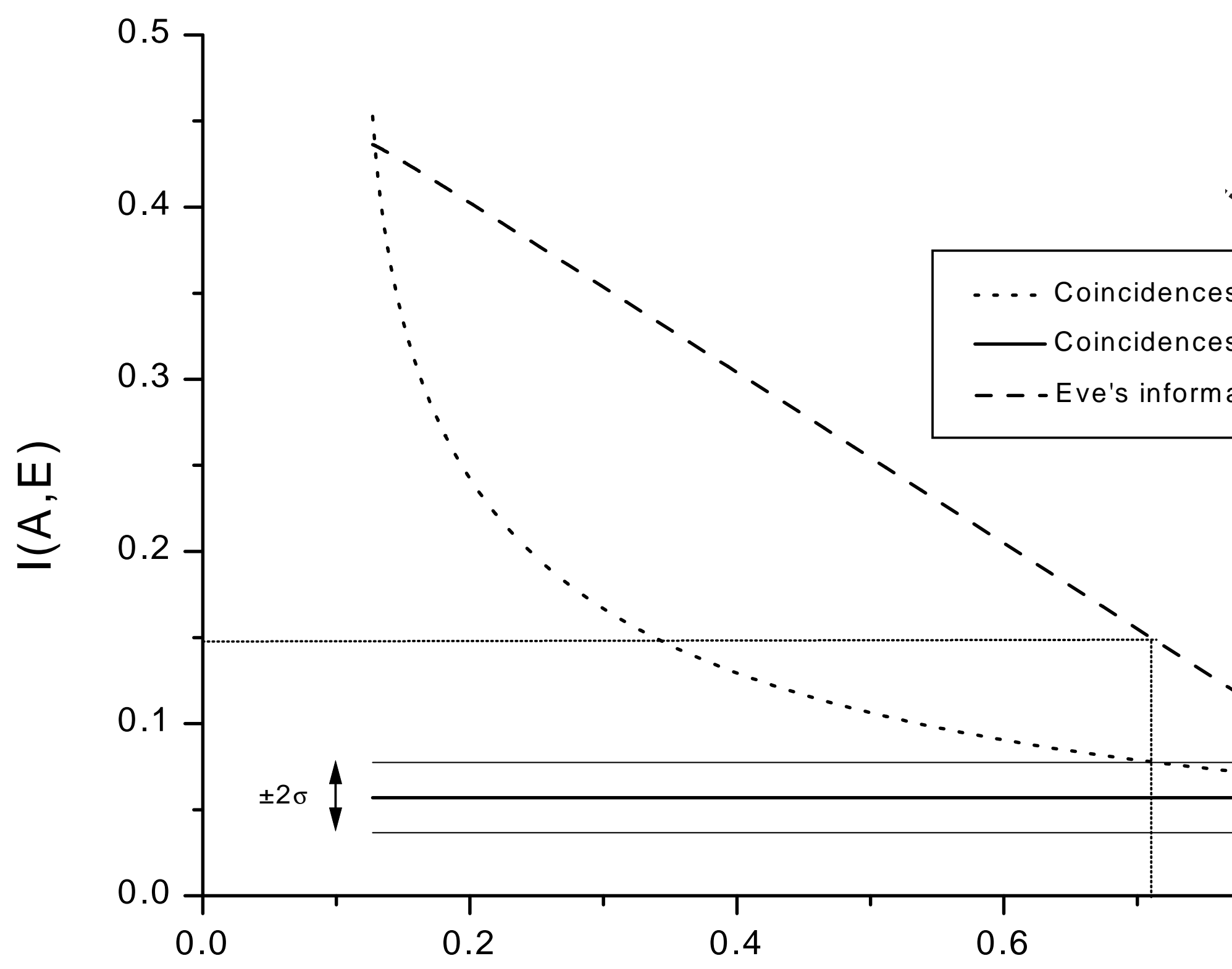




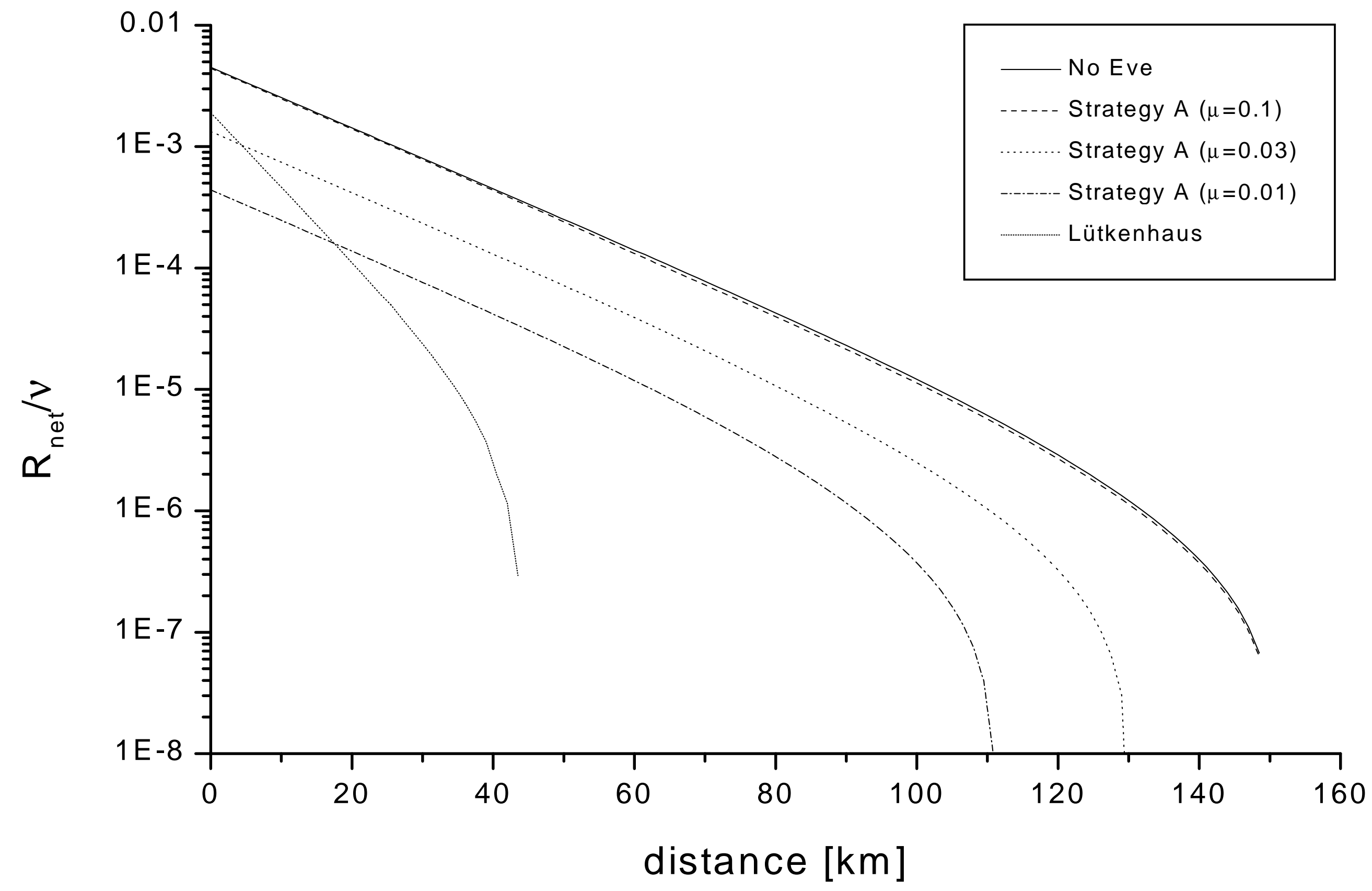




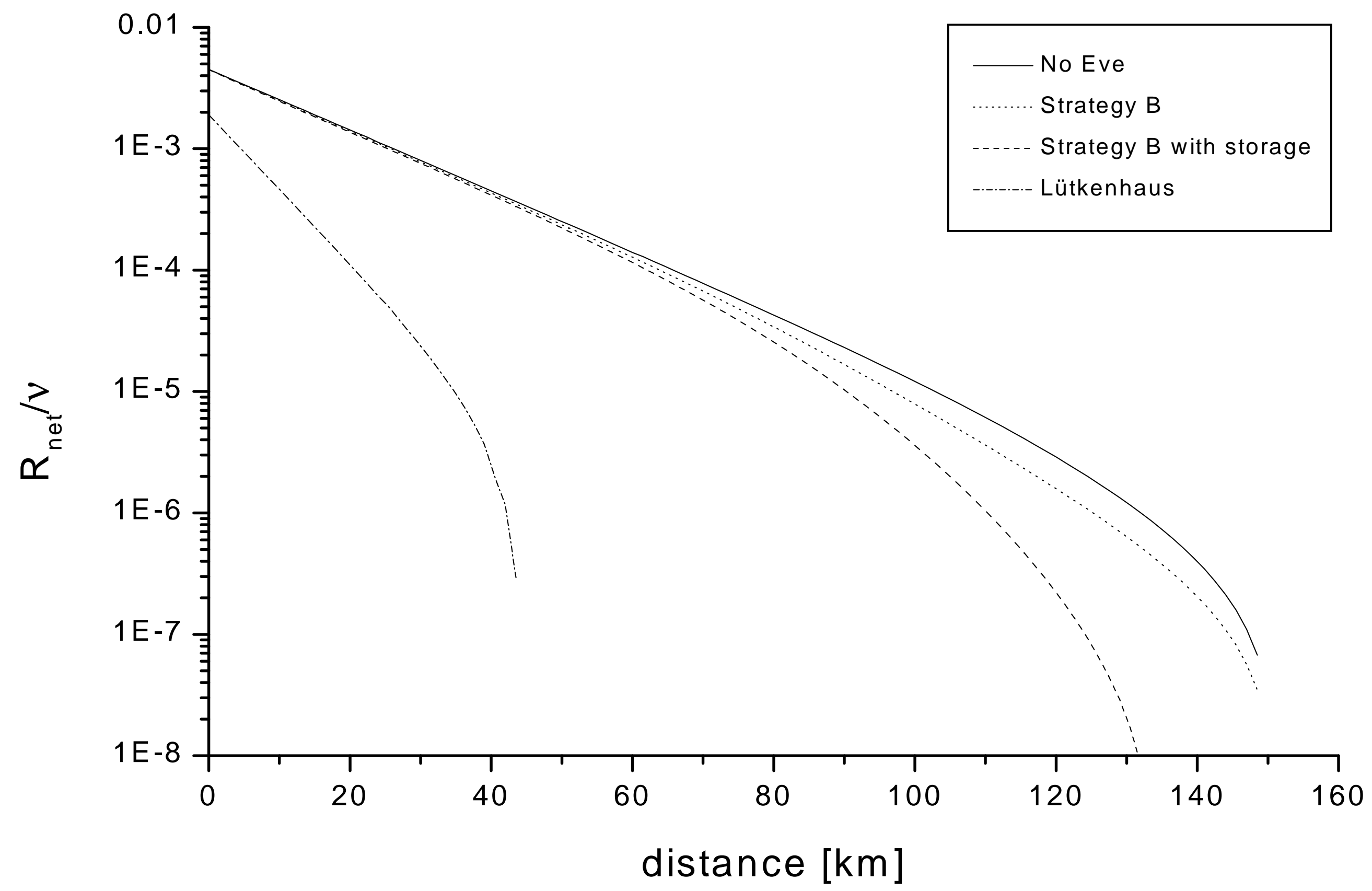

